This item was submitted to Loughborough's Research Repository by the author.

Items in Figshare are protected by copyright, with all rights reserved, unless otherwise indicated.

\title{
A bifurcation study of a dynamic model of a nose landing gear mechanism subjected to external disturbances
}

PLEASE CITE THE PUBLISHED VERSION

http://dx.doi.org/10.2514/6.2015-2653

\section{PUBLISHER}

(C) American Institute of Aeronautics and Astronautics

\section{VERSION}

SMUR (Submitted Manuscript Under Review)

\section{PUBLISHER STATEMENT}

This work is made available according to the conditions of the Creative Commons Attribution-NonCommercialNoDerivatives 4.0 International (CC BY-NC-ND 4.0) licence. Full details of this licence are available at: https://creativecommons.org/licenses/by-nc-nd/4.0/

\section{LICENCE}

CC BY-NC-ND 4.0

\section{REPOSITORY RECORD}

Knowles, James A.C.. 2015. "A Bifurcation Study of a Dynamic Model of a Nose Landing Gear Mechanism Subjected to External Disturbances”. figshare. https://hdl.handle.net/2134/18691. 


\title{
A Bifurcation Study of a Dynamic Model of a Nose Landing Gear Mechanism Subjected to External Disturbances
}

\author{
J. A. C. Knowles*1 \\ ${ }^{1}$ Department of Aeronautical and Automotive Engineering, \\ Loughborough University, UK
}

May 20, 2015

\begin{abstract}
This paper presents a new modelling approach for the analysis of landing gear mechanisms. By replacing the mechanism's rotational joints with equivalent high-stiffness elastic joints, numerical continuation methods can be applied directly to dynamic models of landing gear mechanisms. The effects of using elastic joints are considered through two applications - an overcentre mechanism, and a nose landing gear mechanism. In both cases, selecting a sufficient stiffness for the elastic joint is shown to provide accurate contiuation results. The advantages of this new modelling approach are then demonstrated by considering the unlocking of a nose landing gear with a single uplock/downlock mechanism, when subjected to different orientations and magnitudes of gravitational loading. The unlocking process is shown to be qualitatively insensitive to changes in both load angle and load magnitude, ratifying the robustness of a previouslyproposed control methodology for unlocking a nose landing gear with a single uplock/downlock mechanism.
\end{abstract}

\section{Introduction}

Nonlinear effects often play an important role in governing the dynamic behaviour of engineering systems. A conventional way to analyse these nonlinearities is to perform time history simulations, where the equations of motion are integrated numerically (with respect to time) to determine how the system states evolve. This approach is useful for analysing transient behaviours for one configuration of a system, however it can become time-consuming to investigate the non-transient dynamics (long simulation time), unstable dynamics (re-run simulations for different initial conditions) or multiple system configurations (rerun simulations for each configuration). In such circumstances, it may be useful to use alternative analysis approaches in order to minimise the number and length of any time history simulation runs. One such approach, from the field of dynamical systems theory, is numerical continuation (see $[1,2,3]$ for more information on dynamical systems theory and numerical continuation): here, solutions of interest can be traced out under the simultaneous variation of one or more parameters. The results, when presented in the form of a bifurcation diagram, provide a picture of the invariant dynamic structures that govern the overall system dynamics.

Numerical continuation has been used to analyse a wide range of aeronautical engineering systems, particularly in the areas of nonlinear flight mechanics $[4,5]$ and landing gear shimmy $[6,7,8]$. The ability to identify and compute

*j.a.c.knowles@lboro.ac.uk 
dynamically unstable objects (unstable equilibria or unstable limit cycle oscillations) has been shown to be particularly useful, as such features often separate regions of distinct dynamic behaviour. The use of numerical continuation methods to investigate landing gear mechanisms, however, has been limited due to difficulties associated with performing continuations on constrained dynamic systems. These difficulties arise when a system's dynamics are expressed using more co-ordinates than there are degrees of freedom - for more information, see [9]. To combat these difficulties, previous works expressed the landing gear mechanism as a set of algebraic equations in order to use numerical continuation techniques $[10,11,12]$. Although such an approach was able to demonstrate the benefits that numerical contiunation could have for the design and analysis of landing gear mechanisms, additional dynamic models were required to infer the dynamic stability properties of the equilibria. Furthermore, the models created for the purposes of the continuation runs could not be used to determine any transient dynamic behaviour.

This work presents a new modelling approach that allows numerical continuation methods to be applied to dynamic models of mechanisms. Rather than deal with inelastic constraints (such as planar rotational joints), the method proposed here replaces a sufficient number of these inelastic constraints with high-stiffness elastic joints (such as planar bushes). Replacing the rigid joint constraints with high-stiffness elastic joints increases the mechanism's degrees of freedom, making it possible to apply numerical continuation to a model that can also be used to run time history simulations. The strengths of this new approach are that it works with models that can also produce conventional time history simulations (thus enabling it to be integrated with models created in dynamic simulation software packages, such as simmechanics), and solution stabilities can be determined directly from the continuation algorithm. The methodology is applied to two mechanisms - an overcentre mechanism and a nose landing gear mechanism. The overcentre mechanism model is a set of Ordinary Differential Equations (ODEs), implemented in MATLAB; The landing gear mechanism model used to verify the correctness of the proposed method is also a set od ODEs, implemented in MATLAB; the case study uses a SimMechanics model of a landing gear. For all models, the numerical continuation is performed by the software package AUTO [13], linked to MATLAB via the Dynamical Systems Toolbox [14].

The paper is organised as follows: section 2 introduces the overcentre mechanism model, and demonstrate the new method's region of validity; section 3 introduces the Nose Landing Gear (NLG) model, and considers the new method's applicability to an aerospace application; section 4 investigates the effects of varying gravitational loading on a SimMechanics model of a NLG with a single uplock/downlock mechanism; the final section offers some concluding remarks.

\section{The Overcentre Mechanism}

An overcentre mechanism (Figure 1) is used here to investigate the validity of the proposed approach. This mechanism was chosen because it can be treated analytically with relative ease, and its equilibria contain nonlinear features (in the form of fold bifurcations) that are also present in the more complicated nose landing gear mechanism's equilibria [9, 10]. Furthermore, its simplicity means that the derivation of the equations of motion can be presented in detail in the following subsection, without compromising brevity.

\subsection{Derivation of the Mathematical Model}

The equations of motion are derived by first considering each mechanism link individually as a free body. The forces generated at the mechanism joints are defined to be proportional to the distance between the bodies connected together at that particular point. Considering mechanism link $\mathcal{L}_{1}$ (the link with mass $m_{1}$, 


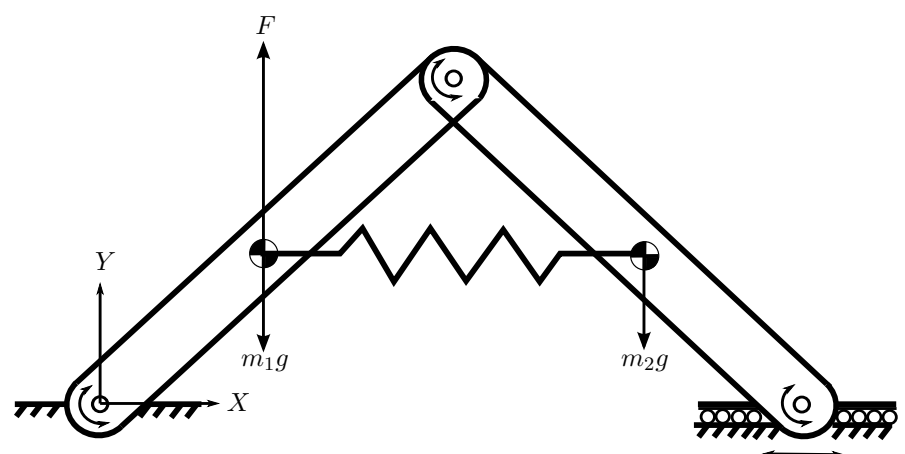

Figure 1: A schematic of the overcentre mechanism.

figure 1), the equations of motion obtained from standard Newtonian mechanics are:

$$
\begin{aligned}
m_{1} \ddot{x}_{1} & =R_{x}^{A}+R_{x}^{B}+F_{s p} ; \\
m_{1} \ddot{y}_{1} & =R_{y}^{A}+R_{y}^{B}-m_{1} g+F ; \\
I_{1} \ddot{\theta}_{1} & =\frac{L_{1}}{2} \sin \theta_{1}\left(R_{x}^{A}-R_{x}^{B}\right)-\frac{L_{1}}{2} \cos \theta_{1}\left(R_{y}^{A}-R_{y}^{B}\right) .
\end{aligned}
$$

Here: $L_{1}$ is the link length; $\ddot{x}_{1}$ and $\ddot{y}_{1}$ are the accelerations of the link's centre of gravity $(\mathrm{cg})$ in the global $X$ and $Y$ directions; $\ddot{\theta}_{1}$ is the angular acceleration of the link about its $\mathrm{cg}$ in the global $\Theta$ direction; $F_{s p}$ is the spring force; the $R$ terms denote internal mechanism reaction forces created at joints $A$ and $B$ in the $X$ and $Y$ directions (as indicated by the subscript and superscript); $n$ dots abve a quantity are used to indicate the $n^{\text {th }}$ derivative of that quantity with respect to time.

The four joint reaction forces are obtained by assuming that a linear, undamped spring acts in each direction at the joints, and that point $A$ co-incides with the origin. As such, the equations for the four internal reaction force terms in equation (1) are:

$$
\begin{aligned}
& R_{x}^{A}=k_{A}\left(x_{1}-\frac{L_{1}}{2} \cos \theta_{1}\right) ; \\
& R_{y}^{A}=k_{A}\left(y_{1}-\frac{L_{1}}{2} \sin \theta_{1}\right) ; \\
& R_{x}^{B}=k_{B}\left(x_{2}-\frac{L_{2}}{2} \cos \theta_{2}-x_{1}-\frac{L_{1}}{2} \cos \theta_{1}\right) ; \\
& R_{y}^{B}=k_{B}\left(y_{2}-\frac{L_{2}}{2} \sin \theta_{2}-y_{1}-\frac{L_{1}}{2} \sin \theta_{1}\right) .
\end{aligned}
$$

The equations of motion for link $\mathcal{L}_{2}$ follow an identical structure to that of equation (1):

$$
\begin{aligned}
m_{2} \ddot{x}_{2} & =-R_{x}^{B}+R_{x}^{C}-F_{s p} ; \\
m_{2} \ddot{y}_{2} & =-R_{y}^{B}+R_{y}^{C}-m_{2} g ; \\
I_{2} \ddot{\theta}_{2} & =\frac{L_{2}}{2} \sin \theta_{2}\left(-R_{x}^{B}-R_{x}^{C}\right)-\frac{L_{2}}{2} \cos \theta_{2}\left(-R_{y}^{B}-R_{y}^{C}\right) .
\end{aligned}
$$

Here, $R_{x}^{C}=0$ (as the joint is prismatic) and $R_{y}^{C}=-k_{C}\left(y_{2}+\frac{L_{2}}{2} \sin \theta_{2}\right)$.

The spring force, $F_{s p}$ contains linear stiffness and damping terms, and is expressed in terms of the horizontal positions and velocities of each link's centre of gravity: 


$$
F_{s p}=c_{s p}\left(\dot{x}_{2}-\dot{x}_{1}\right)+k_{s p}\left(x_{2}-x_{1}-l_{u s}\right),
$$

where $c_{s p}$ is the spring damping, $k_{s p}$ is the spring damping and $l_{u s}$ is the unstrained spring length.

The equations of motion for the overcentre mechanism are therefore described by equations (1) and (3). In the following subsection, these equations are implemented in MATLAB and solved using ODE45 to produce time history results, or the Dynamical Systems Toolbox to produce bifurcation diagrams.

\subsection{Overcentre Mechanism Dynamics}

There are several possible discrepancies that could occur in the overcentre mechanism's long-term dynamics as a result of using stiff springs to approximate the joints: if the internal forces at the joints are of a similar order of magnitude to the joint stiffness values, the equilibrium structure may change qualitatively from the inelastic joint case; if the system contains insufficient damping, spurious oscillations may occur. Both of these points could lead to qualitatively incorrect results when conducting the bifurcation analysis. The purpose of this subsection is to show that these potential discrepancies can be avoided by selecting an appropriate joint stiffness, and to investigate when (and how) the long term dynamics may change.

\subsubsection{Effects of damping on mechanism response}
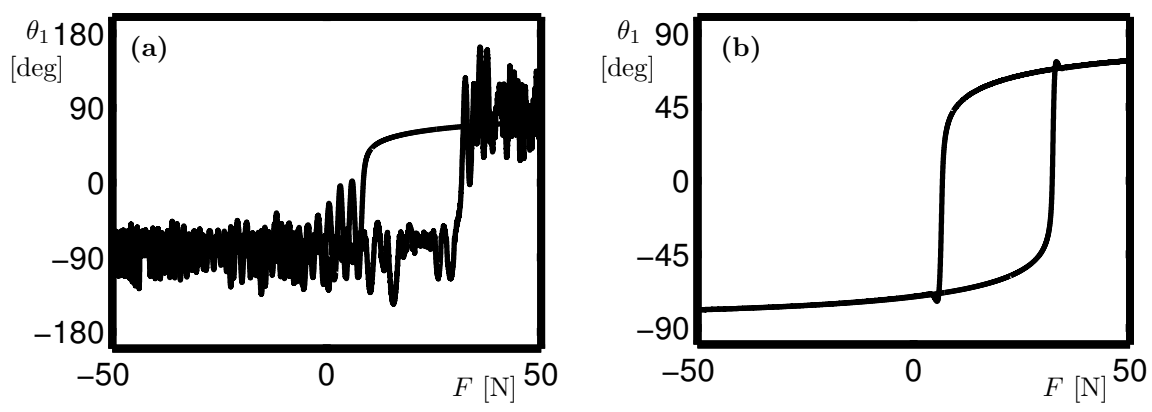

Figure 2: Effect of overcentre spring damping on the transient dynamics of the mechanism.

The transient dynamic response will (of course) be influenced by the level of damping. Figure 2 shows two time history simulation results: panel (a) shows a lightly damped case $(c=0.01 \mathrm{Nm} / \mathrm{s})$; panel (b) shows a heavily damped case $(c=10 \mathrm{Nm} / \mathrm{s})$. The external force $F$ was described by the equation $F=50 \cos (t)$, and run for a simulation time of 360 seconds so that the force could complete a cycle from $+50 \mathrm{~N}$ to $-50 \mathrm{~N}$ and back. Whilst there are significant differences between the two cases, a hysteresis loop is present in both. This is because the underlying equilibrium structure remains the same for both cases, so the long-term dynamic response of each system will converge to the same steady-state solution (albeit at a different rate depending on the damping).

Figure 3 shows two bifurcation diagrams for the overcentre mechanism with different overcentre spring damping ( $c$ in equation (4)): the case in panel (a) is for $c=0.01 \mathrm{Ns} / \mathrm{m}$; the case in panel (b) is for $c=10 \mathrm{Ns} / \mathrm{m}$. Solid curves indicate dynamically stable equilibrium solutions, whilst the dashed curves indicate dynamically unstable equilibrium solutions. The black circles indicate fold bifurcations. Despite the significant differences observed for the transient case in Figure 2, the two bifurcation diagrams are identical, which suggests that 

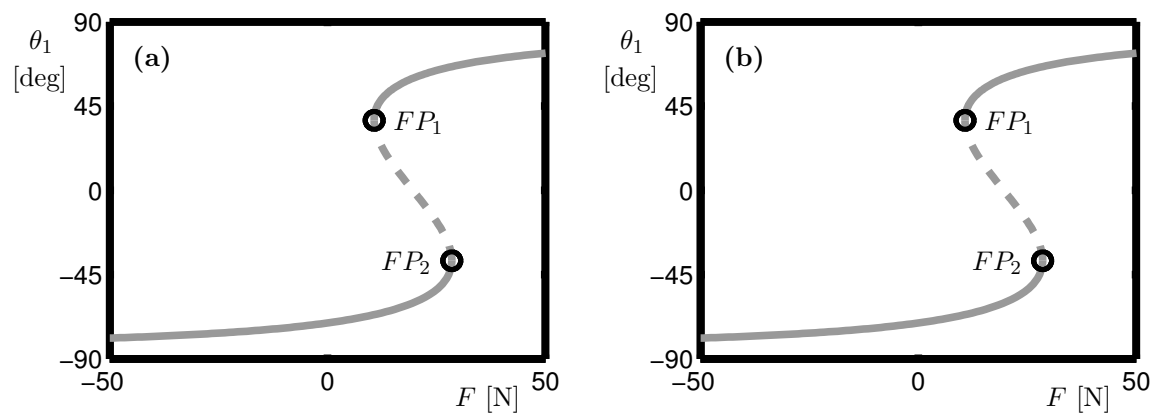

Figure 3: Effect of overcentre spring damping on the underlying dynamics of the overcentre mechanism.

the overcentre mechanism's equilibrium structure is insensitive to changes in damping (provided there is still some damping present within the system).

Previous work [10] explained that the fold bifurcations represent the minimum steady-state force values required for the mechanism to overcentre (i.e. move so that the value of $\theta_{1}$ passes through zero), so it is essential that any bifurcation analysis captures these points correctly. Although changing the spring damping affects the transient response of the overcentre mechanism, the equilibrium structure and fold point bifurcations in figure 3 remain unaffected by changing the system's damping. This suggests that the system damping is not critical when performing a bifurcation analysis of the overcentre mechanism.

\subsubsection{Effects of stiffness on mechanism response}
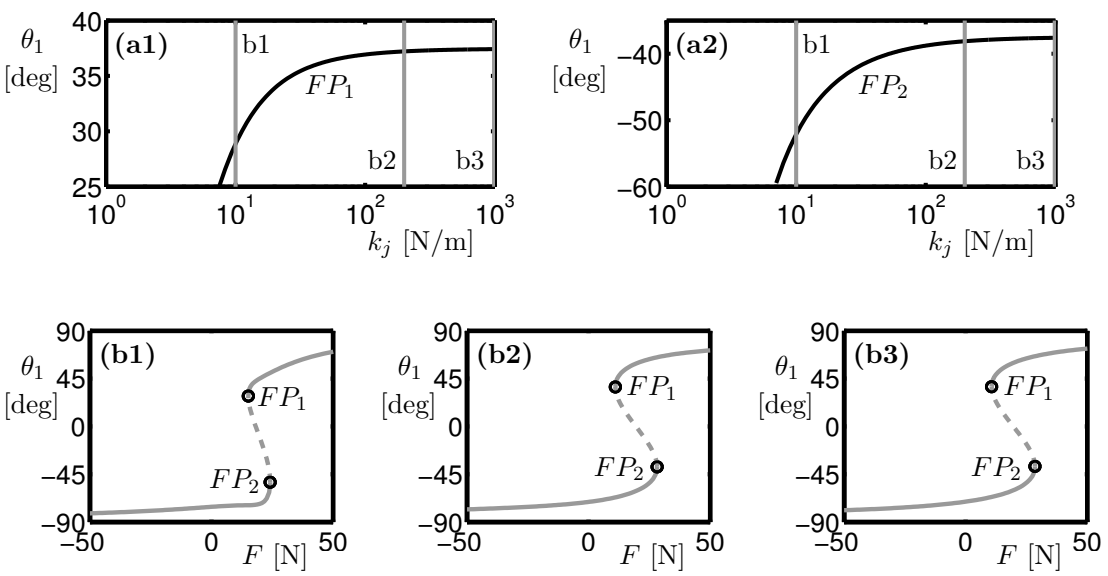

Figure 4: Effect of joint stiffness on the underlying dynamics of the overcentre mechanism.

Figure 4 shows how joint stiffness affects the equilibrium structure of the overcentre mechanism. The angle of fold point bifurcations $F P_{1}$ and $F P_{2}$ is shown as a function of joint spring stiffness is shown in panels (a1) and (a2) by the solid black curve, whilst the vertical grey lines indicate joint stiffness values used to to compute the bifurcation diagrams in panels (b1) - (b3). Unlike the spring damping, the joint stiffness has a direct effect on the fold point bifurcations. As joint spring stiffness is increased, the fold curves in Figure 4(a1) and (a2) level off, tending towards the limit case of a mechanism with inelastic (i.e. infinitely stiff) joints. This shows that it is possible for the numerical continuation results to calculate fold bifurcation locations that are practically 
indistinguishable from the inelastic case, provided a sufficiently high joint stiffness value is chosen.

Panels (b1), (b2) and (b3) in Figure 4 show the angle of link $\mathcal{L}_{1}$ at equilibrium as a function of the externally-applied force $F$, for cases of all joint stiffness values $k_{j}=10,200$ and $1000 \mathrm{~N} / \mathrm{m}$ (respectively): solid curves indicate stable equilibria; dashed curves represent unstable equilibria; black circles indicate fold bifurcations. Considering panels (b1) and (b2) in Figure 4, there are significant quantitative differences that arise due to the change in joint stiffness: the lower branch of equilibria in panel (b1) is quite flat compared to the equivalent section in (b2); the unstable equilibrium curve between the fold points is straighter in (b1) than (b2); the upper branch of equilibria appears to rotate down (about $F P_{1}$ ) when moving from (b1) to (b2). Significantly, however, both results are qualitatively the same, as no additional bifurcations have arisen, nor have any stabilities changed. In contrast, the quantitative differences between results in panels (b2) and (b3) are indistinguishable, suggesting that increasing the joint stiffness from $200 \mathrm{~N} / \mathrm{m}$ to $1000 \mathrm{~N} / \mathrm{m}$ or beyond will not result in a significant increase in the accuracy of the numerical continuation results.

Due to the simplicity of the overcentre mechanism, an analytical solution for its equilibria can be derived. This can then be used to confirm that the process of considering limit point variation is sufficient to determine an appropriate spring stiffness value. Using equilibrium equations derrived in [10], and noting that the applied force here is applied at the $\mathrm{c} / \mathrm{g}$ rather than the joint, the expression for the applied force at equilibrium is:

$$
F=k\left(\frac{L_{1}}{2} \cos \theta_{1}+\frac{L_{2}}{2} \cos \theta_{2}-l_{u s}\right)\left(\tan \theta_{1}-\tan \theta_{2}\right)+m_{1} g+m_{2} g .
$$

Here, $k$ is the spring stiffness, $l_{u s}$ is the unstrained spring length, and all other variables are as outlined in Figure 1.
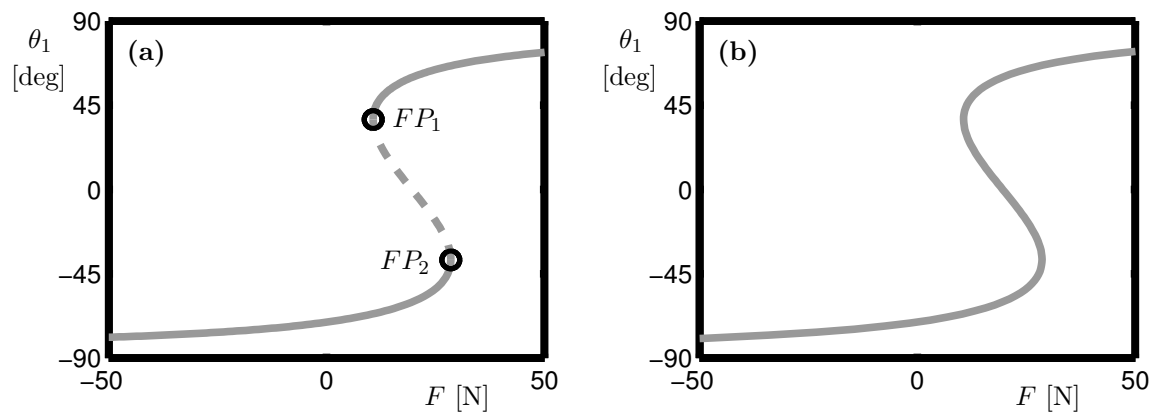

Figure 5: A comparison between numerical continuation result and an analytical solution for the overcentre mechanism.

Figure 5 shows the equilibria for an overcentre mechanism, produced from: (a) the numerical continuation run with joint stiffness $k_{j}=100 \mathrm{~N} / \mathrm{m}$; (b) the analytical solution derived above. The dashed section of the continuation curve in panel (a) represents dynamically unstable equilibria, and black circles indicate fold point bifurcations. Although the computational model used for the continuation run contains an elastic joint, this does not have a significant effect on the qualitative or quantitative structure of the equilibria, as it is identical to the analytical solution in Figure 5(b). This confirms that the addition of an elastic joint does not necessarily compromise the correctness of the solution, provided a sufficient spring stiffness is chosen. It also supports the idea that a sufficient spring stiffness can be identified by considering how points of interest (such as bifurcations) change with joint stiffness - when the joint stiffness no longer causes a significant qualitative of quantitative change, it can be said to be sufficient (for the purposes of providing correct results). 


\section{The Nose Landing Gear Mechanism}

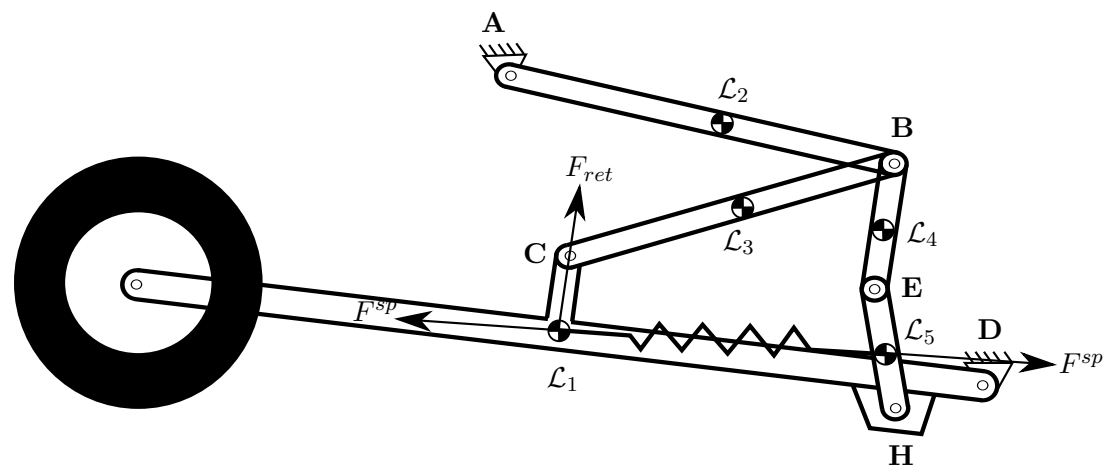

Figure 6: A schematic of the Nose Landing Gear mechanism in the retracted position.

The NLG mechanism (Figure 6) is used here to evaluate the numerical continuation approach in an aerospace engineering application. The following subsections introduce the mathematical model and investigate the range of its validity as joint stiffness and damping is varied.

\subsection{Mathematical Model}

The derivation of the nose landing gear model uses the same approach as that described for the overcentre mechanism. The 15 equations of motion for the NLG mechanism are given below:

$$
\begin{aligned}
m_{1} \ddot{x}_{1}= & R_{x}^{D}-R_{x}^{C}-R_{x}^{F}+m_{6} g_{x}-F_{r e t} \sin \theta_{1}-F_{x}^{s p}+F_{x}^{u l}+m_{1} g_{x} ; \\
m_{1} \ddot{y}_{1}= & R_{y}^{D}-R_{y}^{C}-R_{y}^{F}-m_{6} g_{y}+F_{r e t} \cos \theta_{1}-F_{y}^{s p}+F_{y}^{u l}-m_{1} g_{y} ; \\
I_{1} \ddot{\theta}_{1}= & \frac{L_{1}}{2} \cos \theta_{1}\left(R_{y}^{D}-m_{6} g_{y}\right)-\frac{L_{1}}{2} \sin \theta_{1}\left(R_{x}^{D}-m_{6} g_{x}\right)-\left(F_{x}-x_{1}\right) R_{y}^{H} \\
& +\left(F_{y}-y_{1}\right) R_{x}^{H}-\left(C_{x}-x_{1}\right) R_{y}^{C}+\left(C_{y}-y_{1}\right) R_{x}^{C} ; \\
m_{2} \ddot{x}_{2}= & R_{x}^{B}-R_{x}^{A}+m_{2} g_{x} ; \\
m_{2} \ddot{y}_{2}= & R_{y}^{B}-R_{y}^{A}-m_{6} g_{x} ; \\
I_{2} \ddot{\theta}_{2}= & \frac{L_{2}}{2} \cos \theta_{2}\left(R_{y}^{B}+R_{y}^{A}\right)-\frac{L_{2}}{2} \sin \theta_{2}\left(R_{x}^{B}+R_{x}^{A}\right) ; \\
m_{3} \ddot{x}_{3}= & R_{x}^{C}-R_{x}^{B}+R_{x}^{B b}+m_{3} g_{x} ; \\
m_{3} \ddot{y}_{3}= & R_{y}^{C}-R_{y}^{B}+R_{y}^{B b}-m_{3} g_{y} ; \\
I_{3} \ddot{\theta}_{3}= & \frac{L_{3}}{2} \cos \theta_{3}\left(R_{y}^{C}+R_{y}^{B}-R_{y}^{B b}\right)-\frac{L_{3}}{2} \sin \theta_{3}\left(R_{x}^{C}+R_{x}^{B}-R_{x}^{B b}\right) ; \\
m_{4} \ddot{x}_{4}= & R_{x}^{E}-R_{x}^{B b}+m_{4} g_{x} ; \\
m_{4} \ddot{y}_{4}= & R_{y}^{E}-R_{y}^{B b}-m_{4} g_{y} ; \\
I_{4} \ddot{\theta}_{4}= & \frac{L_{4}}{2} \cos \theta_{4}\left(R_{y}^{E}+R_{y}^{B b}\right)-\frac{L_{4}}{2} \sin \theta_{4}\left(R_{x}^{E}+R_{x}^{B b}\right) ; \\
m_{5} \ddot{x}_{5}= & R_{x}^{H}-R_{x}^{E}+F_{x}^{s p}-F_{x}^{u l}+m_{5} g_{x} ; \\
m_{5} \ddot{y}_{5}= & R_{y}^{H}-R_{y}^{E}+F_{y}^{s p}-F_{y}^{u l}-m_{5} g_{y} ; \\
I_{5} \ddot{\theta}_{5}= & \frac{L_{5}}{2} \cos \theta_{5}\left(R_{y}^{H}+R_{y}^{E}\right)-\frac{L_{5}}{2} \sin \theta_{5}\left(R_{x}^{H}+R_{x}^{E}\right) .
\end{aligned}
$$

All quantities in equation 7 can be identified with reference to figure 6 , noting that $R_{x}^{A}$ is an internal force acting on joint $A$ in the global co-ordinate $x$ direction 
and all other internal forces follow the same naming convention ${ }^{1}$. The terms $H_{x}, H_{y}, C_{x}$ and $C_{y}$ are the $x$ and $y$ co-ordinates of joints $\mathbf{C}$ and $\mathbf{H}$.

Unlike the overcentre mechanism, the spring force is unable to provide damping for all link motions in the NLG mechanism. To produce meaningful time history results, some of the internal joint forces needed to include both stiffness and damping terms. The internal joint forces are therefore given as:

$$
\begin{aligned}
& \mathbf{R}^{\mathbf{A}}=k^{A}\left[\begin{array}{l}
x_{2}-\frac{L_{2}}{2} \cos \theta_{2}-A_{x} \\
y_{2}-\frac{L_{2}}{2} \sin \theta_{2}-A_{y}
\end{array}\right]+c^{A}\left[\begin{array}{c}
\dot{x}_{2}+\frac{L_{2}}{2} \dot{\theta}_{2} \sin \theta_{2} \\
\dot{y}_{2}-\frac{L_{2}}{2} \dot{\theta}_{2} \cos \theta_{2}
\end{array}\right] ; \\
& \mathbf{R}^{\mathbf{B}}=k^{B}\left[\begin{array}{l}
\left(x_{3}-\frac{L_{3}}{2} \cos \theta_{3}\right)-\left(x_{2}+\frac{L_{2}}{2} \cos \theta_{2}\right) \\
\left(y_{3}-\frac{L_{3}}{2} \sin \theta_{3}\right)-\left(y_{2}+\frac{L_{2}}{\sin } \theta_{2}\right)
\end{array}\right]+c^{B}\left[\begin{array}{c}
\left(\dot{x}_{3}+\frac{L_{3}}{2} \dot{\theta}_{3} \sin \theta_{3}\right)-\left(\dot{x}_{2}-\frac{L_{2}}{2} \dot{\theta}_{2} \sin \theta_{2}\right) \\
\left(\dot{y}_{3}-\frac{L_{3}}{2} \dot{\theta}_{3} \cos \theta_{3}\right)-\left(\dot{y}_{2}+\frac{L_{2}}{2} \dot{\theta}_{2} \cos \theta_{2}\right)
\end{array}\right] ; \\
& \mathbf{R}^{\mathbf{B b}}=k^{B b}\left[\begin{array}{l}
\left(x_{4}-\frac{L_{4}}{2} \cos \theta_{4}\right)-\left(x_{3}-\frac{L_{3}}{2} \cos \theta_{3}\right) \\
\left(y_{4}-\frac{L_{4}}{2} \sin \theta_{4}\right)-\left(y_{3}-\frac{L_{3}}{\sin } \theta_{3}\right)
\end{array}\right]+c^{B} b\left[\begin{array}{c}
\left(\dot{x}_{4}+\frac{L_{4}}{2} \dot{\theta}_{4} \sin \theta_{4}\right)-\left(\dot{x}_{3}+\frac{L_{3}}{2} \dot{\theta}_{3} \sin \theta_{3}\right) \\
\left(\dot{y}_{4}-\frac{L_{4}}{2} \dot{\theta}_{4} \cos \theta_{4}\right)-\left(\dot{y}_{3}-\frac{L_{3}}{2} \dot{\theta}_{3} \cos \theta_{3}\right)
\end{array}\right] ; \\
& \mathbf{R}^{\mathbf{C}}=k^{C}\left[\begin{array}{l}
C_{x}-\left(x_{3}+\frac{L_{3}}{2} \cos \theta_{3}\right) \\
C_{y}-\left(y_{3}+\frac{L_{3}}{2} \sin \theta_{3}\right)
\end{array}\right] ; \\
& \mathbf{R}^{\mathbf{D}}=k^{D}\left[\begin{array}{l}
D_{x}-\left(x_{1}+\frac{L_{1}}{2} \cos \theta_{1}\right) \\
D_{y}-\left(y_{2}+\frac{L_{1}}{2} \sin \theta_{1}\right)
\end{array}\right]-c^{D}\left[\begin{array}{l}
\dot{x}_{1}-\frac{L_{1}}{2} \dot{\theta}_{1} \sin \theta_{1} \\
\dot{y}_{1}+\frac{L_{1}}{2} \dot{\theta}_{1} \cos \theta_{1}
\end{array}\right] ; \\
& \mathbf{R}^{\mathbf{E}}=k^{E}\left[\begin{array}{l}
\left(x_{5}-\frac{L_{5}}{2} \cos \theta_{5}\right)-\left(x_{4}+\frac{L_{4}}{2} \cos \theta_{4}\right) \\
\left(y_{5}-\frac{L_{5}}{2} \sin \theta_{5}\right)-\left(y_{4}+\frac{L_{4}}{2} \sin \theta_{4}\right)
\end{array}\right]+c^{E}\left[\begin{array}{l}
\left(\dot{x}_{5}+\frac{L_{5}}{2} \dot{\theta}_{5} \sin \theta_{5}\right)-\left(\dot{x}_{4}-\frac{L_{4}}{2} \dot{\theta}_{4} \sin \theta_{4}\right) \\
\left(\dot{y}_{5}-\frac{L_{5}}{2} \dot{\theta}_{5} \cos \theta_{5}\right)-\left(\dot{y}_{4}+\frac{L_{4}}{2} \dot{\theta}_{4} \cos \theta_{4}\right)
\end{array}\right] ; \\
& \mathbf{R}^{\mathbf{H}}=k^{H}\left[\begin{array}{l}
H_{x}-\left(x_{5}+\frac{L_{5}}{2} \cos \theta_{5}\right) ; \\
H_{y}-\left(y_{5}+\frac{L_{5}}{2} \sin \theta_{5}\right)
\end{array}\right] .
\end{aligned}
$$

Terms in bold are vector quantities, with $\mathbf{R}^{\mathbf{A}}=\left[R_{x}^{A}, R_{y}^{A}\right]^{T}$ (and similarly for all the other joint force vectors). The $k$ terms are the joint stiffness values; the $c$ terms are the joint damping values.

In the following subsection, the equations of motion for the NLG mechanism (equation (7)) are implemented in MATLAB and solved using the Dynamical Systems Toolbox to produce bifurcation diagrams.

\subsection{NLG Mechanism Dynamics}

As with the overcentre mechanism, the introduction of elastic joints into the NLG model has the potential to produce qualitatively incorrect results if insufficient care is taken when choosing joint damping and stiffness values. The following results are presented to convince the reader that appropriate joint damping and stiffness values have been used in the uplocking case study considered - they are not meant to provide an in-depth analysis of the system.

\subsubsection{Effects of joint damping on mechanism response}

Figure 7 shows two bifurcation diagrams for the NLG mechanism around uplock with different joint spring damping values: the case in panel (a) is for $c=1$ $\mathrm{Ns} / \mathrm{m}$; the case in panel (b) is for $c=1000 \mathrm{Ns} / \mathrm{m}$. Solid grey curves indicate dynamically stable equilibrium solutions, whilst the dashed grey curves indicate dynamically unstable solutions. The light grey curves correspond to 'above overcentre' solutions, with the darker grey curves showing 'below overcentre' solutions. The black circles indicate fold bifurcations. As with the overcentre mechanism, the two bifurcation diagrams are identical, which shows that the equilibrium structure is insensitive to changes in damping (provided there is still some damping present). Furthermore, both diagrams capture features relevant to NLG uplocking, as outlined below.

In order to successfully uplock the NLG mechanism, increasing the retraction force $\left(F_{r e t}\right)$ must cause a transition from the above-overcentre curve (light grey) to the below-overcentre curve (dark grey). The case shown in Figure $7(\mathrm{a})^{2}$

\footnotetext{
${ }^{1}$ The exception is the force between links $\mathcal{L}_{3}$ and $\mathcal{L}_{4}$, denoted as $R^{B b}$

${ }^{2}$ or (b) - they are qualitatively the same
} 

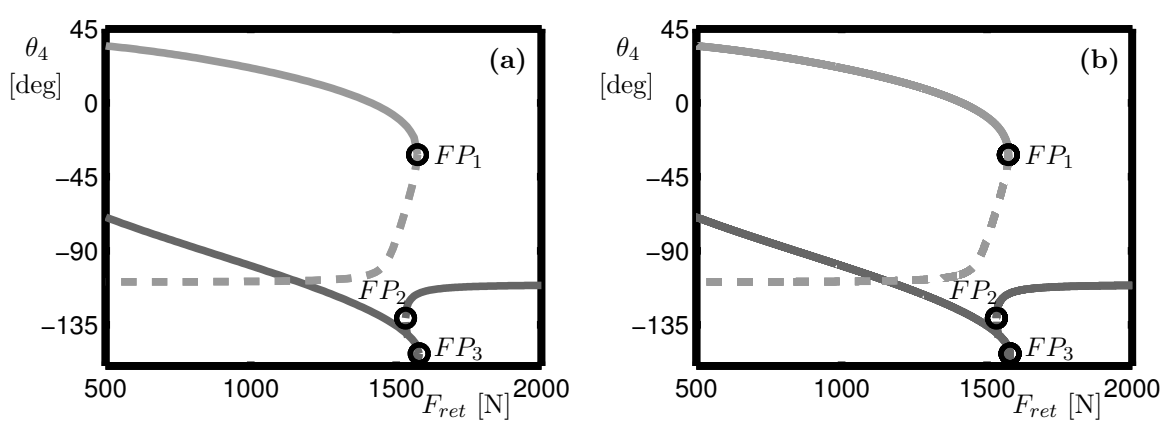

Figure 7: Effect of joint damping on the underlying dynamics of the NLG mechanism.

depicts a landing gear that is able to uplock. The results of Figure 7 show that, as for the overcentre mechanism, the joint damping does not affect $F P_{2}$, or any other aspect of the bifurcation diagram.

\subsubsection{Effects of joint stiffness on mechanism response}

In contrast to the model's insensitivity to joint damping, Figure 8 shows that the bifurcation diagram changes drastically with respect to joint spring stiffness values. Panels (a1) - (a3) show how the angle at which each of the three fold bifurcations ( $F P_{1}$ in (a1), $F P_{2}$ in (a2) and $F P_{3}$ in (a3), black curves) occur, changes as a function of joint stiffness $k_{j}$ (the single value used for all joints). Star points indicate cusp points in the fold curve, whilst grey vertical lines indicate vertical 'parameter slices' for the joint stiffness cases considered in panels (b1) - (b3). Figure 8(a1) and (a3) show that significant qualitative changes occur to fold bifurcations $F P_{1}$ and $F P_{3}$ as joint stiffness is increased: for joint stiffness values of $10^{2} \mathrm{~N} / \mathrm{m}<k_{j}<10^{4} \mathrm{~N} / \mathrm{m}$, vertical 'parameter slices' may intersect each fold curve multiple times, whereas for higher joint stiffness values of $k_{j}>10^{5} \mathrm{~N} / \mathrm{m}$, such vertical 'parameter slices' appear only to intersect each of the fold curves once (for the range of the fold curves that was computed).

In addition to the qualitative changes in the known fold curves, Figure 8(b1) shows that additional fold bifurcations (i.e. ones not necessarily associated with any of the fold curves in Figure 8(a1) - (a3)) also occur at certain stiffness values. The structure of the bifurcation diagram shown in panel (b1) is dominated by joint stiffness effects, and does not capture the correct mechanism behaviour. Increasing the joint stiffness by an order of magnitude leads to a the case shown in panel (b2), which has captured the correct qualitative structure for the landing gear. In fact, further increasing the joint stiffness by another order of magnitude (case in panel (b3)) shows that very little quantitative change occurs for spring stiffnesses $k_{j}>10^{5} \mathrm{~N} / \mathrm{m}$. Due to this, and noting the conclusions from the previous results for the overcentre mechanism, a spring stiffness of $k_{j}=10^{6}$ is thought to provide sufficient accuracy for use with numerical continuation methods.

\section{Landing Gear Unlocking Investigation}

A modern retractable landing gear requires locking mechanisms to fix its position in one of two states: when the aircraft is in contact with the ground, the landing gear needs to be locked in its fully deployed position, or downlocked; when the aircraft is flying, the landing gear needs to be locked in its fully retracted position, or uplocked. Conventional landing gear designs use two different mechanisms to downlock and uplock the landing gear. The landing gear mechanism considered in this section is unusual because it uses a single 

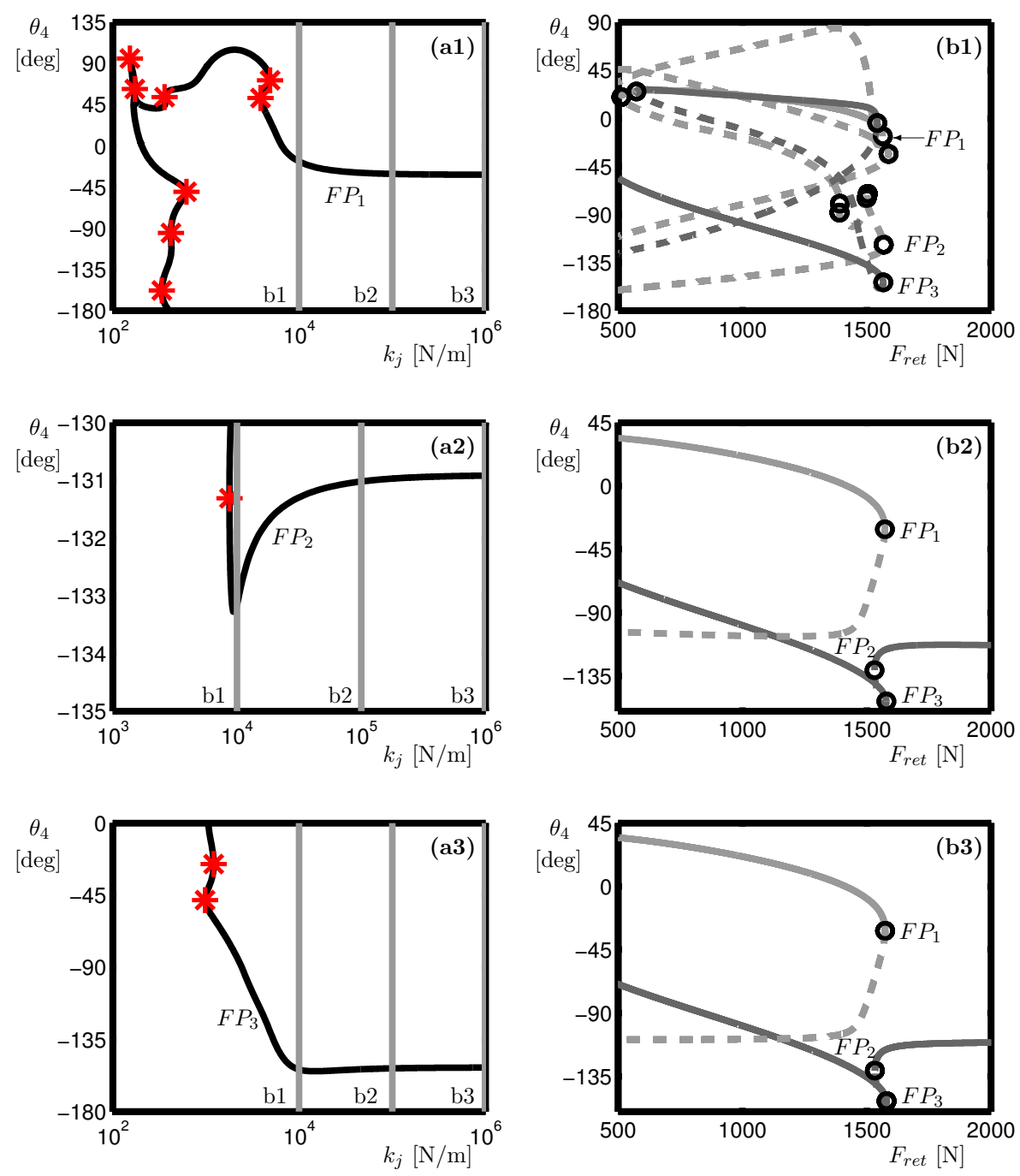

Figure 8: Effect of joint stiffness on the underlying dynamics of the NLG mechanism.

mechanism to both downlock and uplock the nose landing gear.

To demonstrate the potential of the new modelling approach introduced in the previous sections, this section considers a nose landing gear with a single uplock/downlock mechanism, which is modelled in SimMechanics. Dynamic simulation packages, such as SimMechanics, are widely used in the aerospace industry, so a case study of a model created in such a package provides a more realistic demonstration of how numerical continuation may actually be used in an industrial design context. Figure 9 provides a schematic of the SimMechanics model, alongside a side view of the landing gear mechanism produced in the graphic visualiser. Due to the way SimMechanics formulates the equations of motion, only two joints needed to be replaced with bushes (highlighted in the figure) - this resulted in a system of 5 position states with 5 degrees of freedom.

Previous work has already considered the challenge of unlocking this unusual nose landing gear from uplock [9]. The results showed that the conventional control method (using a position measure to define when the mechanism is unlocked) causes the retraction and unlock actuator forces to couple. This coupling means that the actuator forces work against one another once the mechanism reaches overcentre, so some additional force scheduling control is needed to unlock the landing gear. The solution proposed to prevent this coupling is to use a force measure, rather than a position measure, to define when the landing gear 

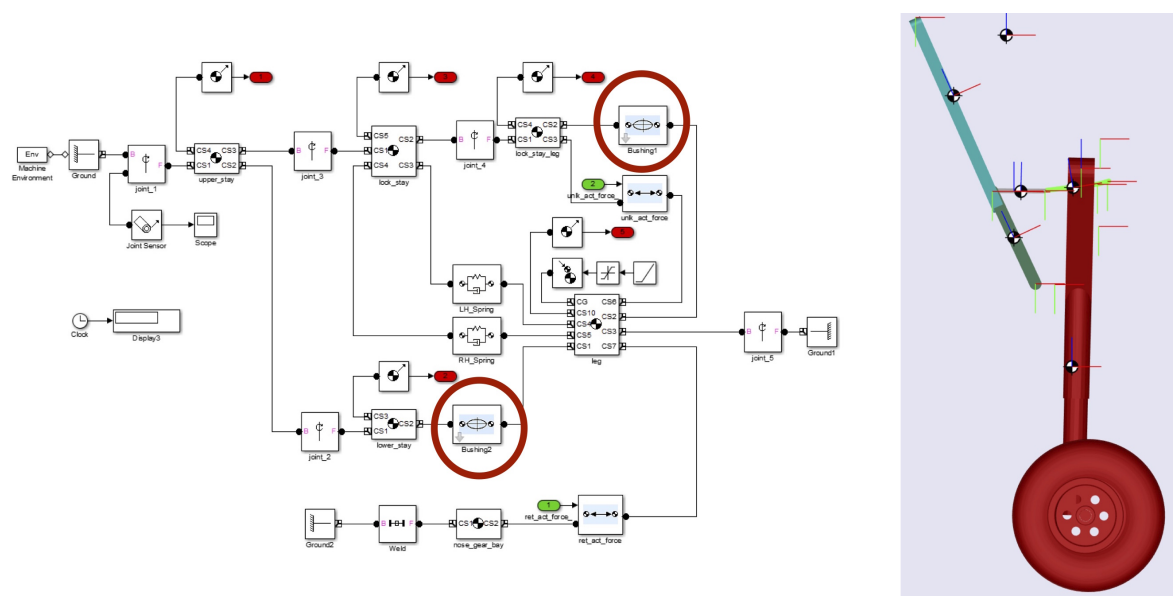

Figure 9: SimMechanics model schematic.

is unlocked [9].

In this section, the robustness of previously-obtained results is considered for the case of an aircraft subjected to external disturbances (such as gust loading). This is achieved by conducting a bifurcation analysis for different gravitational magnitudes (representative of the aircraft experiencing an acceleration) and directions (representative of a change in flight path angle in the vertical plane). Although gust loading is an entirely transient phenomenon, valuable insight into the effects gusts could have on landing gear unlocking can still be gained by considering changes in the underlying equilibrium structures, which govern the dynamics.

\subsection{Robustness of Mechanism Response to Changes in Gravitational Loading Angle}
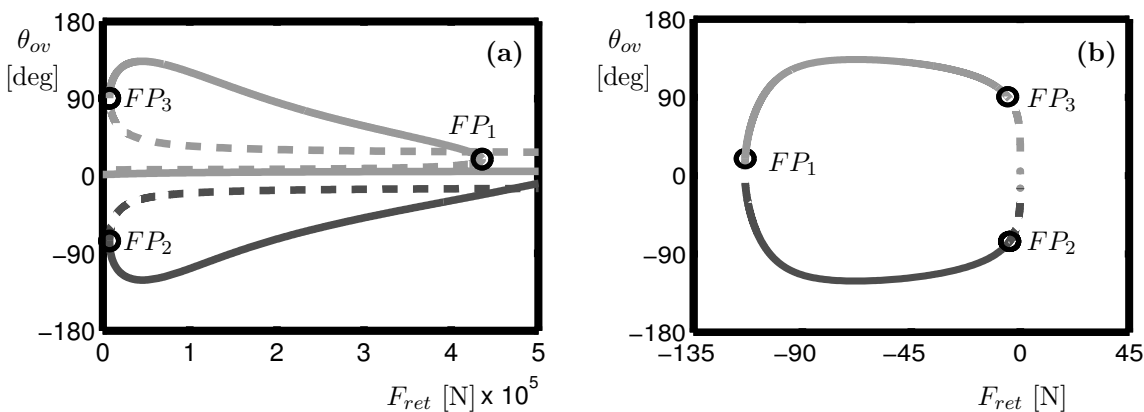

Figure 10: Equilibrium structure for the case of an aircraft in straight and level flight

Figure 10 shows the equilibrium structure for the case of an aircraft in straight and level flight. Panel (a) shows the equilibria in terms of overcentre angle $\theta_{o v}$ (equal to the angle between the two locklinks minus $180^{\circ}$ ) and retraction actuator force $F_{r e t}$; panel (b) shows the same equilibria but in terms of $\theta_{\text {ov }}$ and $\theta_{1}$ (the angle of the shock strut with respect to the vertical axis). In both panels (a) and (b), light grey curves correspond to 'above-overcentre' solutions and dark grey curves show 'below-overcentre' solutions. Dashed curves indicate unstable equilibria, with solid curves used to denote stable equilibria. Fold point bifurcations are represented by black circles. The crucial fold point can be identified as $F P_{1}$, because this point dictates the transition from the above-overcentre curve to the below overcentre curve around the uplock point 
(identifiable as occuring at a large, negative value of $\theta_{1}$ ). The other two fold points in the bifurcation diagram are associated with downlocking of the landing gear, so for clarity of presentation the following analysis will only consider variations in the equilibria around the uplock point. With reference to Figure $10(\mathrm{~b})$ and (a), this means only equilibria that occur when $\theta_{1}<-45^{\circ}$ will be considered, for a retraction force window of $F_{\text {ret }}>3 \times 10^{5} \mathrm{~N}$. The result of this clearer presentation is seen in Figure 11(a2) below, which shows the same case as presented in figure 10.
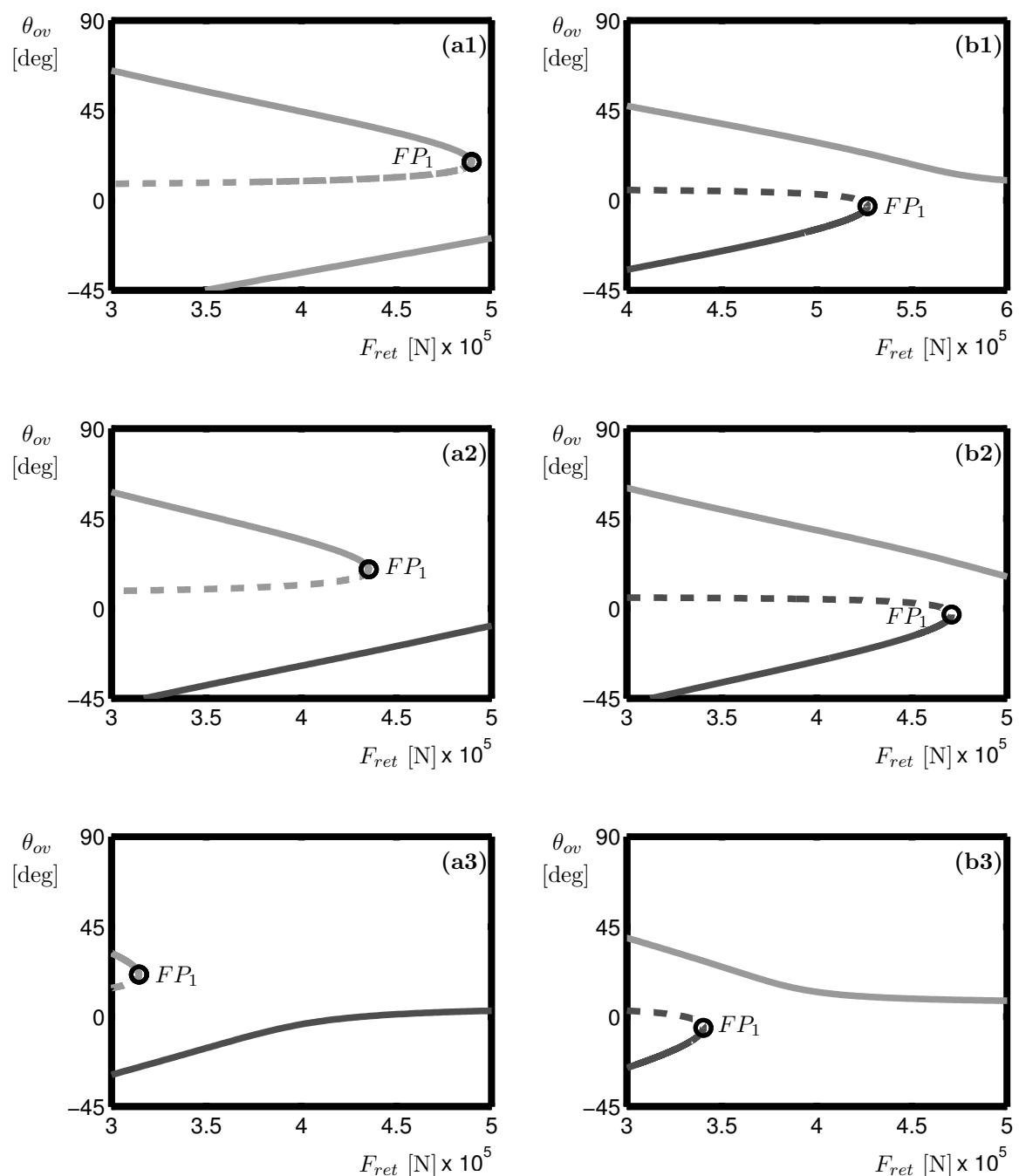

Figure 11: Variation in crucial fold point with changing unlock actuator force $(\mathrm{a}-\mathrm{b})$ and aircraft attitude $(1-3)$.

Figure 11 considers how the crucial fold point, $F P_{1}$, changes as the mechanism is unlocked, for different aircraft attitudes. Aircraft attitudes are modelled by changing the angle at which gravity acts, herein referred to as the gravity angle. Panels (a) show the case with zero unlock force; panels (b) with an unlock force of $500 \mathrm{~N}$. Case 1 is for an aircraft descending steadily at $20^{\circ}$ (gravity angle of $-20^{\circ}$ ); case 2 is for an aircraft in straight and level flight (gravity angle of $0^{\circ}$ ); case 3 is for an aircraft climbing steadily at $20^{\circ}$ (gravity angle of $+20^{\circ}$ ). As before, light grey curves correspond to 'above-overcentre' solutions and dark grey curves show 'below-overcentre' solutions. Dashed curves indicate unstable equilibria, with solid curves used to denote stable equilibria. Fold point bifurcations are represented by black circles.

The first trend to note is that, in all three cases, the effect of the unlock 
actuator is to move $F P_{1}$ from the above-overcentre curve to the below-overcentre curve (compare panels (a) to (b)). Previous work identified that this movement means that the landing gear can be unlocked from uplock[9]: as this movement occurs for several different aircraft attitudes, the movement of $F P_{1}$ is a result that is qualitatively robust with respect to changing aircraft attitude.

The second trend to note is that increasing aircraft gravity angle from $-20^{\circ}$ (descending, panels (1)) to $+20^{\circ}$ (climbing, panels (3)) causes the retraction actuator force at the fold point to decrease. This is because changing the aircraft attitude changes the component of the gravitational acceleration that opposes retraction, so less force is required to uplock the gear on an aircraft descending than for an aircraft that is climbing.

From a design perspective, the implication of these two trends is that the required retraction actuator force for unlocking the gear from uplock would need to be computed for the largest anticipated descent angle. Whilst consideration of the distinct cases in Figure 11 suggests that the fold point's existence is robust to changes in external loading direction, to confirm the extent of this robustness it is necessary to consider when (and how) the qualitative transition occurs from uplocked (panels (a), Figure 11) to unlocked (panels (b), Figure 11).

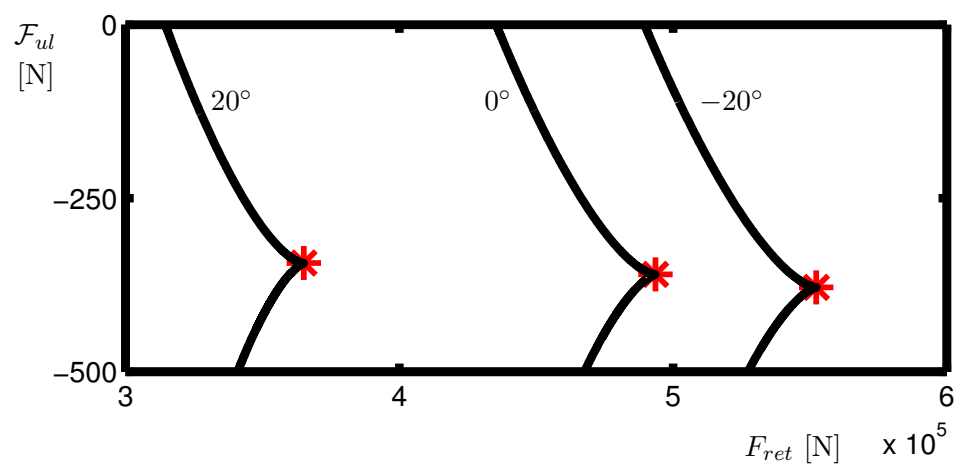

Figure 12: Two-parameter continuation of fold point $F P_{1}$ for gravity angles of $-20^{\circ}, 0^{\circ}$ and $+20^{\circ}$.

Figure 12 presents results from a two-parameter continuation of the fold point $F P_{1}$ for the three aircraft attitude cases considered previously. Previous work identified that the cusp point in the fold curve, indicated by stars in Figure 12, separates the uplocked and unlocked states: for unlock actuator force $\left(\mathcal{F}_{u l}\right)$ values smaller (in magnitude) than at the cusp points, the equilibria structure is qualitatively identical to the cases in Figures 11(a) (i.e. the gear is uplocked, and cannot be unlocked); for unlock actuator force $\left(\mathcal{F}_{u l}\right)$ values larger (in magnitude) than at the cusp points, the equilibria structure is qualitatively identical to the cases in Figures 11(b) (i.e the gear can be unlocked once $F_{\text {ret }}$ values are increased past the cusp point).

Although changing the gravitational angle results in a significant change in required retraction actuator force to unlock the NLG, its effect on the required unlock actuator force is less pronounced. As with the result identified from Figure 11, the limit case that would need to be considered when designing the landing gear is that for a gravity angle of $-20^{\circ}$, however the difference between this limiting case and the other gravitational angle extreme $\left(+20^{\circ}\right)$ is less than a $10 \%$ change in force (compared to almost $35 \%$ change in retraction actuator force). From a design perspective, these results show that the unlock actuator force measure (i.e. the measure used to define when the gear is unlocked) would therefore need to be determined by considering the extremity of the flight envelope that corresponds to a forward-acting acceleration (giving a large, negative gravitational angle). By giving additional consideration to the effects of changing load factor on NLG unlocking, it is possible to identify the critical design case for the mechanism. 


\subsection{Mechanism Response Under Vertical Loading With Non-Unity Load Factor}
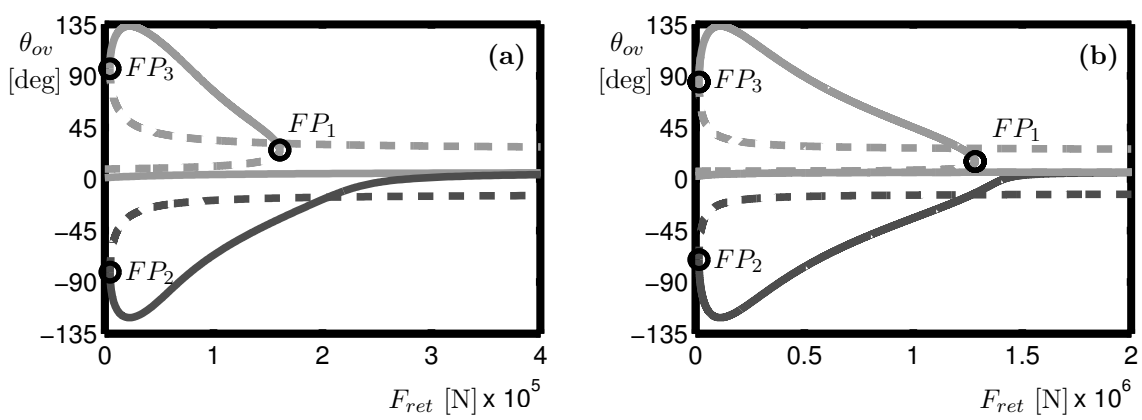

Figure 13: Equilibrium structure for (a) $0.5 \mathrm{~g}$ and (b) $2.5 \mathrm{~g}$ load cases.

Figure 13 compares two vertical (i.e. gravitational angle of $0^{\circ}$ ) loading cases: panel (a) shows the case for the nose landing gear subjected to $0.5 \mathrm{~g}$ loading; panel (b) shows the case for the nose landing gear subjected to $2.5 \mathrm{~g}$ loading. As before, light grey curves correspond to 'above-overcentre' solutions and dark grey curves show 'below-overcentre' solutions. Dashed curves indicate unstable equilibria, with solid curves used to denote stable equilibria. Fold point bifurcations are represented by black circles.

As with changing gravitational angle, only a quantitative change occurs with increasing load factor - the equilibrium structure is stretched out from panels (a) to (b), with equivalent features appearing at higher retraction actuator force values. The increase in load factor also changes the angle, $\theta_{o v}$, at which the three fold points occur. This is because the fold points occur when the moment from the retraction force equals the moment created by the combined weight of the mechanism's elements (shock strut, side stays and lock links), so increasing the loading changes the gear position at which the two moments balance. For the case of the crucial unlock fold point $F P_{1}$, the landing gear reaches a 'more retracted' position before the retraction moment is able to overcome the opposing weight moment. This is also the reason why the retraction force value at $F P_{1}$ increases by more than a factor of 5 from panel (a) to (b) - the retraction force needs to retract the landing gear further before the retraction moment balances the weight moment.

This result indicates that the required retraction actuator force (for unlocking the gear from uplock) would need to be computed for the largest anticipated load factor. As in the previous subsection, to confirm the extent of the robustness of previous results with respect to changing load factor, it is necessary to consider when (and how) the qualitative transition occurs from the uplocked to unlocked positions.

Figure 14 shows three loci of fold points $F P_{1}$ in terms of the two continuation parameters $\left(\mathcal{F}_{u l}\right.$ vs. $\left.F_{r e t}\right)$ at the three different gravity angles considered in the previous subsection, for a load factor of 0.5. Cusp points are indicated by stars. The variations in fold locus with gravitational angle are proportionally similar to the variations observed in Figure 12 - the limiting case for both unlock actuator and retraction actuator force is $-20^{\circ}$, and the unlock force at the cusp points increases slightly as gravity angle is decreased.

In comparison to the $0.5 \mathrm{~g}$ loading case considered in Figure ??, the results for the 2.5g loading case (Figure 15) show that the unlock force at the cusp point now varies significantly between the three gravity angle cases considered, however the maximum unlock actuator force required is still determined by the $-20^{\circ}$ case. The variation between the three unlock force values at the cusp points in Figure 15 is counter-intuitive when comparing the $20^{\circ}$ and $0^{\circ}$ with the equivalent curves in Figure 14: the higher load case requires a lower unlock force 


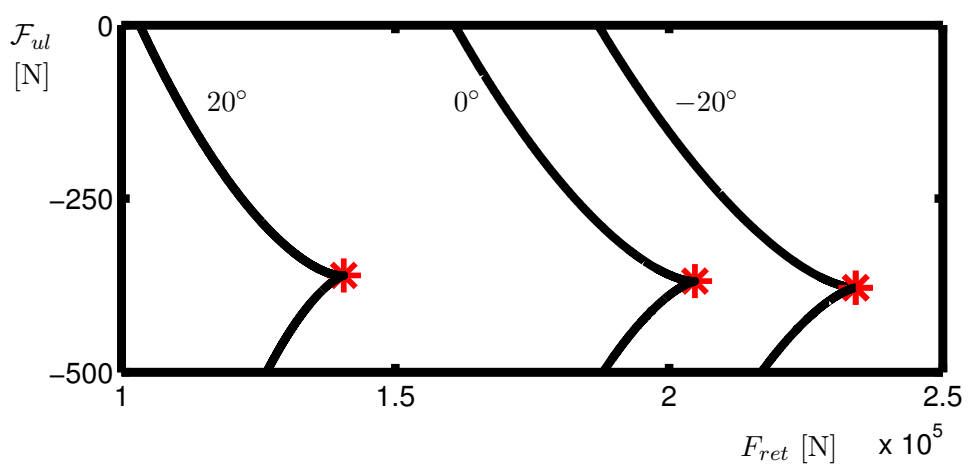

Figure 14: Two-parameter continuation of fold point $F P_{1}$ for gravity angles of $-20^{\circ}, 0^{\circ}$ and $+20^{\circ}-0.5 \mathrm{~g}$ load case.

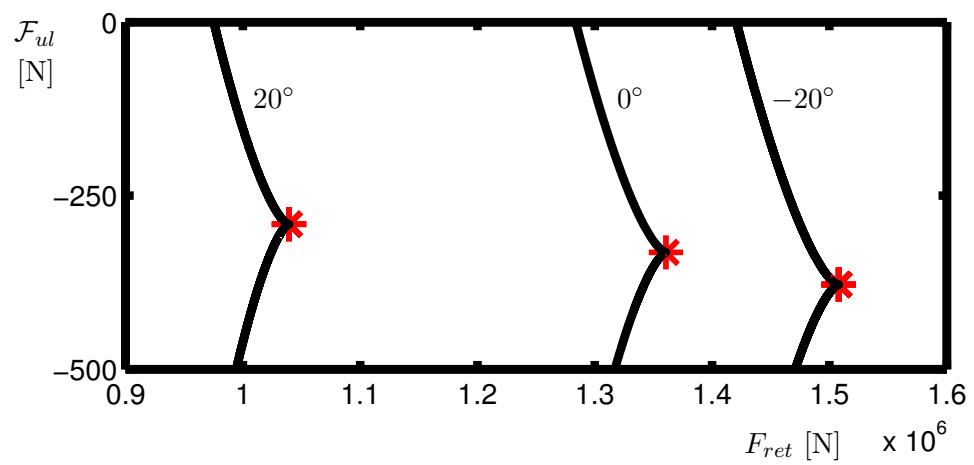

Figure 15: Two-parameter continuation of fold point $F P_{1}$ for gravity angles of $-20^{\circ}, 0^{\circ}$ and $+20^{\circ}-2.5 \mathrm{~g}$ load case.

to reach the cusp points. The reason for this counter-intuitive variation is due to the geometry of the landing gear at uplock: with positive gravitational angles (or small negative angles), gravity aids unlocking from uplock by working to pull the lock links away from the unlocked position; based on the results obtained here, gravity angles of around $-20^{\circ}$ or lower would have the opposite effect, i.e. gravity would work to uplock the landing gear (or work against unlocking from uplock). This result highlights the importance of correct identification of critical load cases, which will vary from one landing gear design to the next.

\section{Concluding Remarks}

This paper proposes a new modelling approach to enable numerical continuation to be applied directly to dynamic models of mechanisms. By using elastic joints within the mechanism, the continuation software package AUTO was able to trace out equilibrium solutions and bifurcations under the simultaneous variation of one or more parameters. The effects of replacing inelastic joints with elastic equivalents was evaluated by considering mathematical models of an overcentre mechanism and a nose landing gear mehcanism. Both models were shown to be insensitive to changes in system damping, however when low stiffness values were used in the elastic joints qualitative and quantitative changes were observed. Two-parameter continuation runs were subsequently conducted to ensure that the chosen joint spring stiffness values produced qualitatively correct results.

The new modelling approach was then used to investigate the nose landing gear unlocking process. The purpose of this study was to determine if a 
previously-proposed unlocking strategy for a particular NLG with a single uplock/downlock mechanism is robust with respect to changes in external loading conditions. The effect of changes in aircraft orientation was captured by changing the direction in which gravity acts within the model. Whilst significant qualitative changes occurred in the equilibrium structure, the critical fold point was shown to remain qualitatively robust with respect to these changes. A two-parameter continuation study supported the robustness of previous results, confirming that the use of a force measure in the unlocking process could be operated robustly with respect to changes in aircraft attitude.

The effect of load factor variations on unlocking was investigated by considering how the crucial cusp point changed as load factor was increased. It was found that higher load factors required lower unlock actuator force values to unlock the landing gear for two of the three angle cases considered, and had little noticeable effect for the third angle case. This counter-intuitive result was attributed to the geometry of the landing gear, as gravity acts to aid the locklinks when unlocking from uplock. Further studies would be needed to investigate the combined effects of gravitational angle and load factor in more depth, however the results from the case study presented in this paper suggest that a critical design case would arise for an aircraft pitching nose-down under high positive $g$ loading.

Future work could also look to implement a force-measure control strategy in dynamic simulations of a landing gear unlocking mechanism with external disturbances, in order to evaluate the effectiveness of this proposed unlocking strategy in a transient situation. The change in equilibrium structure may also be of interest to mathematicians, potentially warranting an in-depth mathematical analysis of some of the behaviours introduced in this paper.

Overall, this study has introduced a novel approach to modelling mechanisms and used this to evaluate the robustness of a force measure in the unlocking of a nose landing gear with a single uplock/downlock mechanism.

\section{References}

[1] Strogatz, S., Nonlinear dynamics and chaos, Springer, 2000.

[2] Guckenheimer, J. and Holmes, P., Nonlinear Oscillations, Dynamical Systems and Bifurcations of Vector Fields, Applied Mathematical Sciences Vol. 42, Springer, 1983.

[3] Krauskopf, B., Osinga, H. M., and Galàn-Vioque, J., Numerical Continuation Methods for Dynamical Systems, Springer, 2007.

[4] Goman, M., Zagainov., G. and Khramtsovsky, A., Application of bifurcation methods to nonlinear flight dynamics problems, Progress in Aerospace Sciences, 33:539-586, 1997.

[5] Thompson, J. and Macmillen, F., Nonlinear flight dynamics of high performance aircraft, Philosophical Transactions of the Royal Society of London A, 356(1745):2163-2333, 1998.

[6] Thota, P., Krauskopf, B. and Lowenberg, M. H., Interaction of Torsion and Lateral Bending in Aircraft Nose Landing Gear Shimmy, Nonlinear Dynamics, 57(3), 2009.

[7] Thota, P., Krauskopf, B. and Lowenberg, M. H., Multi-parameter bifurcation study of shimmy oscillations in a dual-wheel aircraft nose landing gear, Nonlinear Dynamics, 70(2) (2012), pp. 1675-1688

[8] Howcroft, C., Krauskopf, B., Lowenberg, M. H., and Neild, S. A., Influence of variable side-stay geometry on the shimmy dynamics of an aircraft dualwheel main landing gear, SIAM Journal on Applied Dynamical Systems, 12(3) . (2013), pp. 1181-1209. 
[9] Knowles, J.A.C., Krauskopf, B., Lowenberg, M.H. and Neild, S.A., A bifurcation study to guide the design of a landing gear with a combined uplock/downlock mechanism, Proceedings of the Royal Society A: Mathematical, Physical and Engineering Sciences, Vol. 470, No. 2172, December 2014, pp. 1-23.

[10] Knowles, J.A.C., Krauskopf, B. and Lowenberg, M.H., Numerical Continuation Applied to Landing Gear Mechanism Analysis, AIAA Journal of Aircraft, Vol. 49, No. 8, July - August 2011, pp. 1254-1262.

[11] Knowles, J. A. C., Krauskopf, B. and Lowenberg, M. H., Neild, S. A. and Thota, P., Numerical Continuation of a Three-dimensional Main Landing Gear Mechanism, Nonlinear Dynamics, 79(1) (2013), pp. 331-352.

[12] Knowles, J. A. C., Krauskopf, B. and Lowenberg, M. H., Neild, S. A. and Thota, P., Numerical Continuation Analysis of a Dual-Sidestay Main Landing Gear Mechanism, Journal of Aircraft, Vol. 51, No. 1, Jan 2014, pp. 129-143.

[13] Doedel, E., Champneys, A., Fairgrieve, T., Kuznetsov, Y., Sandstede, B., and Wang, X., AUTO 97 : Continuation and bifurcation software for ordinary differential equations, http://indy.cs.concordia.ca/auto/, May 2001.

[14] Coetzee, E.B., Krauskopf, B. and Lowenberg, M.H., Dynamical Systems Toolbox for MATLAB, https://github.com/ecoetzee/Dynamical-SystemsToolbox, July 2011.

[15] Thota P, Krauskopf B, Lowenberg MH., Multi-parameter bifurcation study of shimmy oscillations in a dual-wheel aircraft nose landing gear, Nonlinear Dyn. 70 (2012), pp. 1675-1688. 